ARTICLE OPEN

\title{
High-throughput discovery of high Curie point two-dimensional ferromagnetic materials
}

\author{
Arnab Kabiraj $\mathbb{D}^{1 凶}$, Mayank Kumar ${ }^{1}$ and Santanu Mahapatra ${ }^{1}$
}

Databases for two-dimensional materials host numerous ferromagnetic materials without the vital information of Curie temperature since its calculation involves a manually intensive complex process. In this work, we develop a fully automated, hardwareaccelerated, dynamic-translation based computer code, which performs first principles-based computations followed by Heisenberg model-based Monte Carlo simulations to estimate the Curie temperature from the crystal structure. We employ this code to conduct a high-throughput scan of 786 materials from a database to discover 26 materials with a Curie point beyond $400 \mathrm{~K}$. For rapid data mining, we further use these results to develop an end-to-end machine learning model with generalized chemical features through an exhaustive search of the model space as well as the hyperparameters. We discover a few more high Curie point materials from different sources using this data-driven model. Such material informatics, which agrees well with recent experiments, is expected to foster practical applications of two-dimensional magnetism.

npj Computational Materials (2020)6:35; https://doi.org/10.1038/s41524-020-0300-2

\section{INTRODUCTION}

The recent experimental demonstration of ferromagnetism in twodimensional (2D) materials: $\mathrm{Crl}_{3}{ }^{1}$ and $\mathrm{Cr}_{2} \mathrm{Ge}_{2} \mathrm{Te}_{6}{ }^{2}$ at low temperatures, has opened a new horizon of nanotechnology research since these materials inherit the potential to revolutionize engineering fields like spintronics ${ }^{3}$, valleytronics ${ }^{4}$, sensing and memory technologies ${ }^{5}$. In their classical work, Mermin and Wanger $^{6}$ showed that under an isotropic Heisenberg model, long-range magnetic order must be absent in 2D. However, the more recent discovery of even room-temperature ferromagnetism in monolayer $\mathrm{VSe}_{2}{ }^{7}$ and $\mathrm{MnSe}_{2}{ }^{8}$ has been possible as the strong magnetocrystalline anisotropy of these 2D materials lifts the Mermin-Wanger restriction. So far, a plethora of 2D ferromagnetic (FM) materials ${ }^{9-14}$ have been computationally predicted, including a few general-purpose 2D materials databases ${ }^{15-17}$ containing hundreds to thousands of entries. However, none of these databases contain the most crucial parameter for 2DFM materials relevant for practical applications: the transition temperature or Curie point $\left(T_{\mathrm{C}}\right)$. This is due to the fact that the computational determination of $T_{C}$ is a highly complex process, which involves a manual heuristics-based search for the ground-state and lowenergy spin configurations. Identification of different magnetic exchanges (direct, super or double) within the neighbouring atoms and mapping them appropriately in a Monte Carlo based spin-flipping simulator has also been a manually intensive exercise. The choice of the model Hamiltonian (Ising instead of Heisenberg) used to simulate the spin-flipping with temperature, also raises a question on the reliability of the Curie temperatures of 2D materials predicted so far in the literature ${ }^{10,14}$.

Recently, an algorithm ${ }^{18}$ has been proposed which can search and predict the collinear, experimentally verified ground and lowenergy spin states for bulk materials, almost optimally and exhaustively. Building on this, we develop a code, which performs first principles-based computations followed by Heisenberg model-based Monte Carlo simulations to predict the Curie point accurately from any magnetic 2D material crystal structure.
Software engineering on this code makes it capable to execute such rigorous calculations in a high-throughput manner, even on a workstation-grade computer with GPU (graphical processing unit) acceleration. We use this code to determine the Curie points of materials from a suitable database ${ }^{16}$. To our surprise, almost $47 \%$ of the 786 materials classified as FM, turned out to be antiferromagnetic (AFM) upon close inspection by our code. The $T_{C}$ and other magnetic properties could be successfully determined for 157 materials, among which 26 materials reveal beyond $400 \mathrm{~K}$ Curie point. Close agreement with experimentally measured $T_{C}$ for a few materials validates our high-throughput methodology. In pursuit of faster discovery of high- $T_{C}$ materials, we further develop a machine-learning (ML) pipeline using these 157 data points. Using this $\mathrm{ML}$ model, we identify a few high $T_{C}$ 2DFM materials from the literature and other databases.

The informatics, which optimally balances the rigorousness and efficiency, gives us unprecedented opportunity to compare the magnetic properties of a very large number of materials with diverse structures, which may lead to many new insights on $2 \mathrm{D}$ magnetism. For example, we understand why the inclusion of the higher-order neighbours is important for $T_{C}$ calculation for certain materials and why it is not for the others. We observe several violations of the Goodenough-Kanamori ${ }^{19,20}$ rules for superexchange, the origin of which is open for further exploration. We also demonstrate that a machine-learning model can capture the complex process of temperature-dependent spin-flipping with exceptional accuracy. Our work thus significantly upgrades the computational materials toolbox to foster practical applications of two-dimensional magnetism.

\section{RESULTS}

High-throughput computational framework

We first explain the workflow of our automated code as illustrated in Fig. 1. The unit cell of the material is first fed to a recently developed module ${ }^{18}$ of the open-source python library

\footnotetext{
${ }^{1}$ Nano-Scale Device Research Laboratory, Department of Electronic Systems Engineering, Indian Institute of Science (IISc) Bangalore, Bangalore 560012, India.

凶email: kabiraj@iisc.ac.in
} 


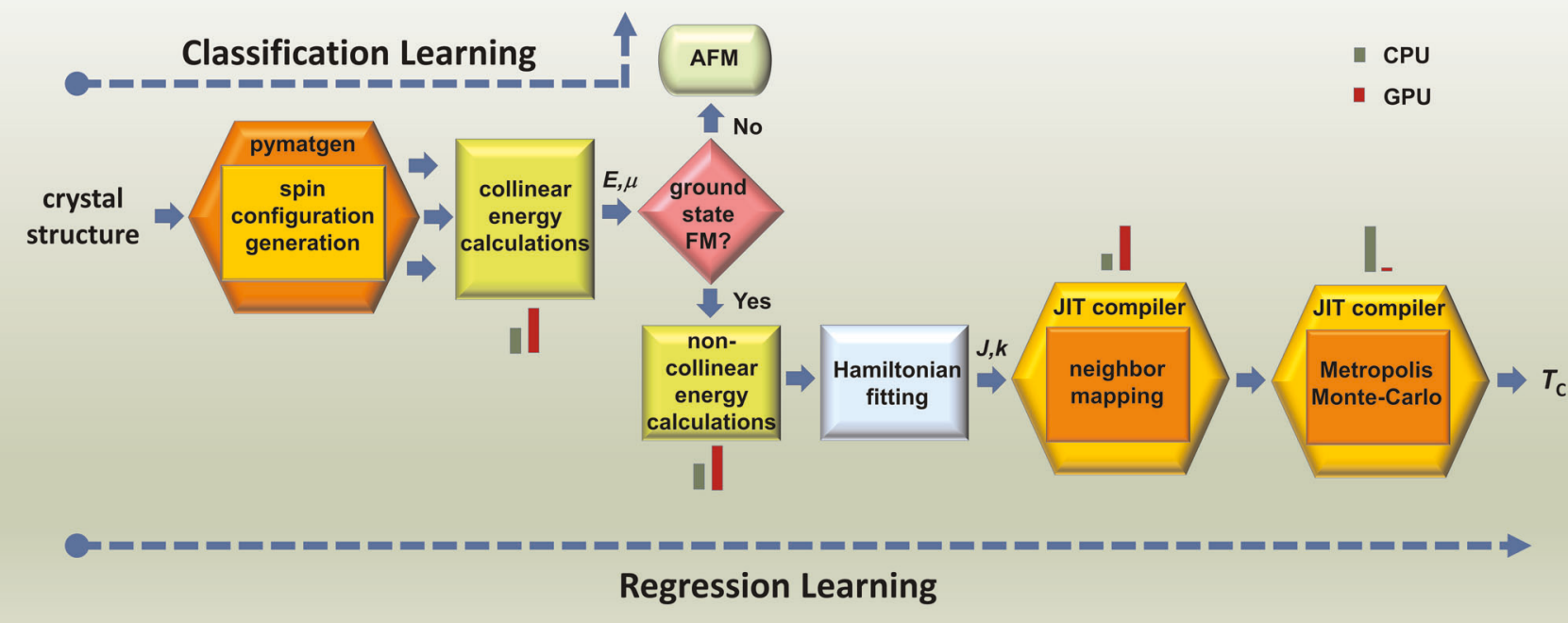

Fig. 1 Automated high-throughput machinery. The different building blocks of our end-to-end code for determining properties of 2DFM materials along with an estimation of CPU and GPU usage of each block.

pymatgen $^{21}$, which generates different FM and AFM spin configurations of the material based on symmetry analysis. Eliminating the heuristics-based approach, pymatgen not only helps to automate the process of the $T_{C}$ calculation, but also makes it more rigorous and thus reliable [see Methods]. The codegenerated spin configurations for experimentally synthesized materials $\mathrm{Crl}_{3}{ }^{1}$ and $\mathrm{Cr}_{2} \mathrm{Ge}_{2} \mathrm{Te}_{6}{ }^{2}$ and newly predicted material $\mathrm{Cr}_{3} \mathrm{Te}_{4}{ }^{10}$ are shown in Supplementary Figs. $1-3$ as examples. These structures are then relaxed using collinear density functional theory with Hubbard correction (DFT $+U$ ) and their energies are calculated. At this stage, if the ground state is found to be AFM, the material is discarded. Here we also calculate the magnetic moment $(\mu)$ of each atom of the structure. Since the magnetocrystalline anisotropic energy (MAE) is essential for the existence of long-range magnetic order in 2D materials, it is calculated using non-collinear DFT including the effects of spin-orbit coupling (SOC) in the next step. These calculations also reveal the easy magnetization axis (EMA) of the 2DFM material, which can be important for specific applications. All these calculations provide us enough information to fit the DFT energy values to the following Heisenberg Hamiltonian:

$$
\begin{aligned}
H= & -\frac{1}{2} \sum_{i, j} J_{1} \boldsymbol{S}_{i} . \boldsymbol{S}_{j}-\frac{1}{2} \sum_{i, I} J_{2} \boldsymbol{S}_{i} . \boldsymbol{S}_{I}-\frac{1}{2} \sum_{i, m} J_{3} \boldsymbol{S}_{i} \cdot \boldsymbol{S}_{m}-\frac{1}{2} \sum_{i, n} J_{4} \boldsymbol{S}_{i} \cdot \boldsymbol{S}_{n} \\
& -k_{x} \sum_{i}\left(S_{i}^{x}\right)^{2}-k_{y} \sum_{i}\left(S_{i}^{y}\right)^{2}-k_{z} \sum_{i}\left(S_{i}^{z}\right)^{2} .
\end{aligned}
$$

Here, $J_{1}, J_{2}, J_{3}$ and $J_{4}$ are the nearest-neighbour (N1), 2nd nearestneighbour (N2), 3rd nearest-neighbour (N3) and 4th nearestneighbour (N4) exchange coupling constants and $\boldsymbol{S}_{i}, \boldsymbol{S}_{j}, \boldsymbol{S}_{l}, \boldsymbol{S}_{m}$ and $\boldsymbol{S}_{\boldsymbol{n}}$ are the spins at sites $i, j, l, m$, and $n$, respectively. $k_{x}, k_{y}$, and $k_{z}$ are the magnetic anisotropy constants in the $x, y$ and $z$ directions. $S$ is computed as $\mu /\left(2 \mu_{\mathrm{B}}\right)$, where $\mu$ is the local magnetic moment of the magnetic ions.

For 2D materials, the classical Monte Carlo (MC) based solution of the Heisenberg Model is known to accurately predict the transition temperature ${ }^{22}$. First, the 2DFM unit cell is multiplied to make a supercell large enough to eliminate size effects, and using a GPU accelerated search, all the neighbours of all the sites are mapped into an 1D array structure. This is then used to perform a Heisenberg model-based classical MC simulation using a semicompiled dynamic-translation based module, from which the $T_{\mathrm{C}}$ of the material can be obtained. For a few materials, no in-plane anisotropy is observed which are classified as $\mathrm{XY}$ magnets. Mermin-Wagner theorem ${ }^{6}$ prohibits spontaneous symmetry breaking in these kinds of systems where the spin degree of freedom is $\leq 2$. Instead, XY magnets exhibit a Berezinskii-Kosterlitz-Thouless (BKT) transition to a quasi-long-range ordered low-temperature phase. For these materials, $T_{C}$ is calculated from the following equation obtained from Monte Carlo simulations of the $\mathrm{XY}$ model ${ }^{23}$ :

$$
T_{\mathrm{C}}=\frac{0.89}{8 k_{\mathrm{B}}}\left(E_{\mathrm{AFM}}-E_{\mathrm{FM}}\right)
$$

where $k_{\mathrm{B}}$ is the Boltzmann constant and $E_{\mathrm{FM}}$ and $E_{\mathrm{AFM}}$ are energies of the FM and the most stable AFM solutions normalized by the number of atoms.

Though the Ising model can provide good results for materials with extremely high anisotropy, significant overestimation of $T_{C}$ may happen for materials with moderate to low anisotropy ${ }^{10,11,14}$. The anisotropic Heisenberg model takes care of this problem and effectively balances the contributions between exchange and anisotropy. However, it requires further MAE calculations and the MC simulation becomes computationally much more expensive. Although this is a high-throughput study, leveraging software engineering [see Methods], we decide to use the rigorous Heisenberg model without compromising the accuracy.

\section{Database search}

We identify the database $\mathrm{C}^{2} \mathrm{DB}^{16}$ as the ideal database to conduct our study based on the following reasons. (1) The authors have performed a preliminary classification between FM and AFM materials and a large number (786) of materials are classified as FM. (2) These materials have been explored by a "systematic combinatorial approach" where almost all known layered exfoliable materials are covered ${ }^{15}$, and by substituting the atoms, the authors have predicted a lot of new materials. This kind of variation is ideal to train machine-learning models, which is one of our primary goals. Also, recent synthesis of janus ${ }^{24}$ and other species substituted ${ }^{25} 2 \mathrm{D}$ materials with no bulk analogues have made practical application of this kind of "synthetic" 2D materials possible. (3)The authors have calculated several properties of interest including thermodynamic stability and energy above the convex hull which helps us to estimate the chance of potential 
synthesis of these materials. The electronic structures have also been computed, which tells us about the presence of important properties like half-metallicity.

Interestingly, after a close examination by our code, 368 of the 786 materials classified as FM in the database turns out to be actually AFM. The pymatgen magnetism module explores the symmetry allowed spin-configuration space almost exhaustively, and in the process also explores large AFM supercells, which probably the authors of C2DB could not afford to do in their general-purpose study. A few discrepancies can also arise from the difference in DFT settings between the studies. Analyses of a considerable amount of materials have failed and thus have been discarded due to various computational limitations [see Methods]. Sheet 1 of Supplementary Spreadsheet 1 lists all the examined materials with FM/AFM classification as found by our method. In the end, the $T_{C}$ of 157 2DFM materials could be successfully computed, among which 12 materials are found to be $X Y$ magnets. These materials belong to more than 20 different prototype structures, which are shown in Fig. 2.
Discovery of high- $T_{\mathrm{c}}$ materials

Almost all experimentally synthesized 2D materials are there in C2DB, except $\mathrm{Cr}_{2} \mathrm{Ge}_{2} \mathrm{Te}_{6}$, which too we have included manually in this set. We have not included those 2DFM materials whose ferromagnetism cannot be accurately modelled by the Heisenberg or the $\mathrm{XY}$ model, such as known itinerant material $\mathrm{Fe}_{3} \mathrm{GeTe}_{2}{ }^{26,27}$. Sheet 1 of Supplementary Spreadsheet 2 lists all the calculated properties, as well as the computed Curie temperatures of these 2DFM materials. To include interactions of $\mathrm{N}$ neighbours in the Hamiltonian, $\mathrm{N}+1$ spin configurations are required, and the number of configurations generated by pymatgen is also listed in Supplementary Spreadsheet 2. Given enough configurations, we have included up to the 4th nearest-neighbour interaction in this work. The $T_{C} S$ are calculated using two methodologies: (1) commonly used nearest-neighbour approach: including only the $\mathrm{N} 1$ interaction and fitting the energies of the FM and the most stable AFM configurations, (denoted as 'TC' in the spreadsheet) and (2) multi-neighbour approach: interactions including up to N4 (listed under the 'TC_exact' column). Apparently, the TC_exact

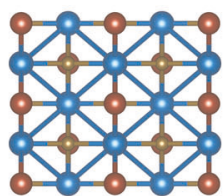

$\mathrm{FeOCl}, \mathrm{FeSe} \& \mathrm{NiO}_{3}$

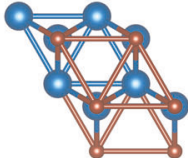

$\mathrm{CH}$

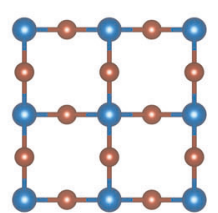

GeS2
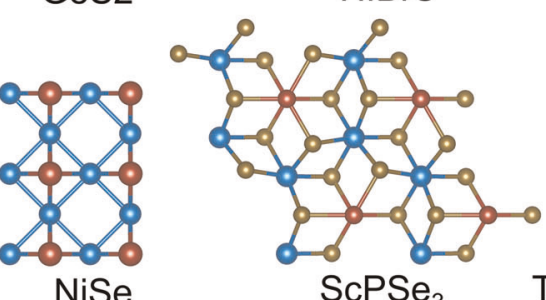

$\mathrm{ScPSe}_{3}$

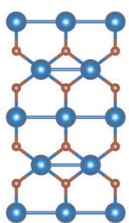

AuSe

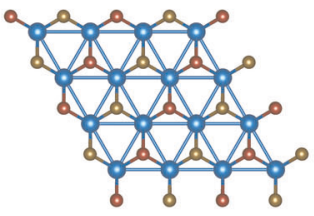

$\mathrm{HfBrS}$

$\mathrm{Bil}_{3}$

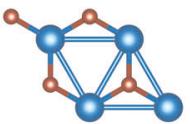

GaS

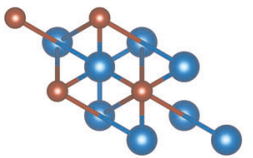

GaSe

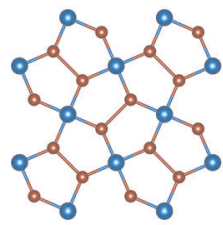

$\mathrm{MnS}_{2}$

$\mathrm{MoS}_{2} \&$ MoSSe

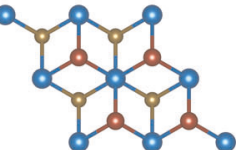

BiTel \& $\mathrm{Cdl}_{2}$

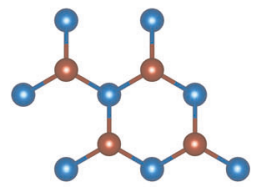
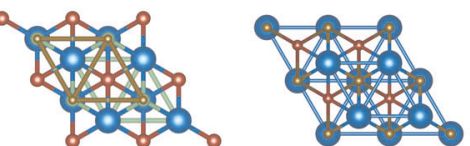

$\mathrm{Ti}_{2} \mathrm{CH}_{2} \mathrm{O}_{2} \& \mathrm{Ti}_{2} \mathrm{CO}_{2} \quad \mathrm{Ti}_{3} \mathrm{C}_{2} \mathrm{O}_{2}$

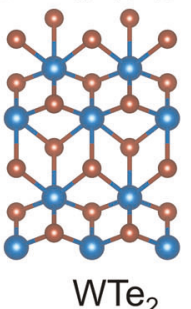

Fig. 2 Prototypes of training data. Top view of prototypes (according to C2DB) of all the 2DFM materials for which $T_{\mathrm{C}}$ could be determined using our method. Blue balls represent the magnetic metal ions and the red and brown balls signify non-magnetic ions. Note that the grouped prototypes, e.g. $\mathrm{FeOCl}$, FeSe, and $\mathrm{NiO}_{3}$ show the same structure cation-wise, but have different numbers of anions. However, all prototypes in such a group look similar from the top. 
should provide a better estimate of the real $T_{\mathrm{C}}$ of the material. However, to our surprise, we observe that TC, which is computationally much economic, is close to TC_exact in most of the cases, except a few as described below. (1) Materials with more than one distinct metal layer (prototypes $\mathrm{CH}, \mathrm{GaSe}, \mathrm{Cdl}_{2^{-}}$ MXene, $\mathrm{Ti}_{2} \mathrm{CO}_{2}$, and $\mathrm{Ti}_{2} \mathrm{CH}_{2} \mathrm{O}_{2}$ ). For prototypes $\mathrm{CH}$ and $\mathrm{GaSe}$, there is no layer containing anions between the metal layers, which gives rise to strong inter-layer direct exchange in addition to the intra-layer superexchange. In prototypes $\mathrm{Cdl}_{2}, \mathrm{Ti}_{2} \mathrm{CO}_{2}$ and $\mathrm{Ti}_{2} \mathrm{CH}_{2} \mathrm{O}_{2}$, which are all $M$ Xenes, the distinct metal layers are connected by anionic layers, where both the inter-layer as well as intra-layer exchange interactions play a pivotal role in deciding the $T_{C}$. Clearly, considering only the $\mathrm{N} 1$ interactions in these materials is not accurate enough, as reflected in the huge differences between TC and TC_exact. In passing, we note that, for a few MXenes, pymatgen could only generate two configurations, thus, the TC value has been repeated as TC_exact. (2) Materials with square or rectangular lattice (prototype $\mathrm{FeOCl}$, FeSe, $\mathrm{GeS}_{2}$ and NiSe). Here, the N1s or N2s are the atoms situated in the diagonally opposite corners of the square or rectangle, where superexchange is expected to be feeble at best and only strong direct exchange could persist. The difference in distance between $\mathrm{N} 1 \mathrm{~s}$ and N2s are also very small in these materials, which again makes the inclusion of the higher neighbour interactions necessary [see Supplementary Figs. 4 and 5]. In a few materials (Janus) the effect of higher-order neighbours is not possible to take into account due to moderate distortion in the lattice and $T_{\mathrm{C}}$ value has been repeated as TC_exact.

The calculated $T_{C}$ (TC_exact) by our automated code for the experimentally synthesized materials $\mathrm{Crl}_{3}{ }^{1}, \mathrm{Cr}_{2} \mathrm{Ge}_{2} \mathrm{Te}_{6}{ }^{2}$, and $\mathrm{MnSe}_{2}{ }^{8}$ matches the experimental reports very closely without any manual tinkering of parameters, validating the generalization and accuracy of our method. For T-phase $\mathrm{VSe}_{2}$ our predicted $T_{\mathrm{C}}$ is only $114.33 \mathrm{~K}$, whereas room temperature ferromagnetism has been reported ${ }^{7}$. However, it must be noted that the authors have reported strong substrate dependence of the magnetism and $T_{\mathrm{C}}$ in this study which explains this apparent discrepancy. Also, our code confirms the magnetism to be in-plane in this material which matches the experimental report. With Supplementary Table 1, Supplementary Note 1 and Supplementary Figs 1, 2, and 6, we explain in detail how our code works for $2 \mathrm{FM}\left(\mathrm{Crl}_{3}{ }^{1}, \mathrm{CrGeTe}_{3}{ }^{2}\right)$ and $2 \mathrm{AFM}\left(\mathrm{FePS}_{3}{ }^{28}, \mathrm{NiPS}_{3}{ }^{29}\right.$ ) experimentally synthesized $2 \mathrm{D}$ materials.

We finally discover a total of 26 materials with $T_{C}>400 \mathrm{~K}$ and 32 materials with $T_{\mathrm{C}} \geq 300 \mathrm{~K}$, making these materials suitable for practical device applications. Interestingly, many of these materials are known to show a "low" amount of magnetism in bulk forms, such as materials containing $\mathrm{Rh}, \mathrm{Ru}, \mathrm{Mo}, \mathrm{W}, \mathrm{Sc}, \mathrm{Ti}$, and $\mathrm{Zr}$, which were ignored in previous heuristics-based searches ${ }^{10}$. However, our study suggests that the materials containing the above-mentioned metals can indeed show a "decent" (0.59-3.96 $\mu_{\mathrm{B}}$ /atom) magnetic moment in $2 \mathrm{D}$ crystal form along with high- $T_{C}$, possibly because of the enhanced electron localization. Also, for some of these materials the anisotropy is not great, but the difference in energy between the FM and AFM states, which ultimately translates to exchange parameters, helps to lift the $T_{\mathrm{C}}$ beyond the room-temperature.

Since the magnetic properties of these materials can greatly depend on the value of $U$ (Hubbard Correction), we also calculate the $T_{C}$ of the 26 promising high- $T_{C}$ materials with much more accurate material-specific $U$ valuess ${ }^{30}$ [see Methods] and present the results in Sheet 3 of Supplementary Spreadsheet 2. Apart from a single material (MolN_Pmmn), we observe that the $T_{C}$ of the rest of the materials remains either close to $400 \mathrm{~K}$ or becomes much higher than that. We also noticed in some cases the $T_{\mathrm{C}}$ value has been significantly enhanced with the application of these tailormade $U$ values. Thus, we expect that some of the materials whose $T_{C}$ values fall in the $250-400 \mathrm{~K}$ range might exhibit much higher $T_{\mathrm{C}}$ if it is calculated using material-specific $U$.

\section{Machine-learning model}

Due to an increasing amount of available data, machine-learning has recently found many applications in the field of solid-state materials science ${ }^{31}$. Very recently, training on about 2500 experimentally reported Curie temperature of bulk materials, accurate $\mathrm{ML}$ models to predict $T_{\mathrm{C}}$ of bulk materials have been developed $^{32}$. 2D magnetism is fundamentally different from the magnetism of the bulk materials as most of the time anisotropy does not play such a significant role there. Therefore, In this emerging field, one doesn't have the luxury of a sufficient amount of data points to train on. Based on the 157 data points obtained from our database-search we develop a machine-learning model to predict the $T_{\mathrm{C}}$ from the crystal structures. To decide the best model and features, we use the autoML library automatminer (https://github.com/hackingmaterials/automatminer). This tool takes structures as input and decorates the dataset with easily computable and chemically and physically meaningful features ${ }^{33}$. Then the dataset is cleaned and reduced and is sent to the autoML library TPOT $^{34}$, which stochastically searches the model and the hyperparameter space using a genetic algorithm and finds the best model for the given dataset. After this extensive search [see Methods], we find an excellently fitted pipeline with average crossvalidation (CV) score $94.57 \mathrm{~K}^{2}$, which is reported as the mean square error (MSE) on the training set. For the FM/AFM classification problem, we also try to find a suitable pipeline using the same method, with our examined 525 FM + AFM data points. The fitted pipeline reports an average CV score of a lowly $72.89 \%$ (accuracy) on the training set, which is understandable considering the complexity of the problem and the size and skewness of the data.

To test the generalization and predictive power of these $\mathrm{ML}$ pipelines, we construct a test set from reported 2DFM materials. Also, quite a few selected materials are included in the test set from a separate database ${ }^{17}$ with completely new structures and complex compositions. After inspecting these materials using our code, we find a few materials to be AFM which have been claimed as FM. This discrepancy can originate from the use of different DFT settings. This, along with fitting with a large number of spin configurations also causes a difference in fitted $J$ values as observed for few materials (see Supplementary Fig. 3). Details of 22 materials identified as FM are provided in Sheet 2 of Supplementary Spreadsheet 2. The new prototypes encountered in this set are illustrated in Fig. 3.

Figure $4 a$ illustrates the accuracy of the ML pipeline prediction against the DFT-MC calculated $T_{C}$ for all the train and test data. The ML predicted $T_{C}$ is also listed in the 'TC_exact predicted' column of Supplementary Spreadsheet 2. The distribution of absolute errors in train and test data have been plotted in Fig. $4 \mathrm{~b}$. Although the pipeline has fitted to train data with high accuracy, generalization to test data does not seem so well. The MSE value also turns out to be $30335.46 \mathrm{~K}^{2}$. Although the small train data size could be partially responsible for this, the main reason is possibly the introduction of unseen crystal structures. For instance, although we have not trained with even a single material containing $\mathrm{La}$, the prediction for $\mathrm{LaBr}_{2}$ is exceptionally close probably because we had a lot of crystals with similar structures in our train data. The same argument can be applied to $\mathrm{Mn}_{2} \mathrm{H}_{2} \mathrm{NO}_{2}$ and $\mathrm{Cr}_{3} \mathrm{Te}_{4}$. The ML classifier pipeline has also been tried on the test data containing a total of $123 \mathrm{FM}+$ AFM samples, which yields a decent $73.17 \%$ accuracy. Sheet 2 of Supplementary Spreadsheet 1 tabulates all the materials tried and their ultimate fate as well as the classification prediction.

During this exercise, we identify $\mathrm{CrO}_{2}{ }_{2} \mathrm{P} 4 / \mathrm{mmm}$ and $\mathrm{ZnNi}_{2} \mathrm{O}_{5}$ as high- $T_{C}$ materials, while the claim of $\mathrm{Cr}_{3} \mathrm{Te}_{4}$ possessing high- $T_{C}$ has also been verified, albeit the Curie temperature turns out to be much lower than the reported Ising model predicted value ${ }^{10}$, but higher than the experimentally reported value for bulk $\mathrm{Cr}_{3} \mathrm{Te}_{4}$. 

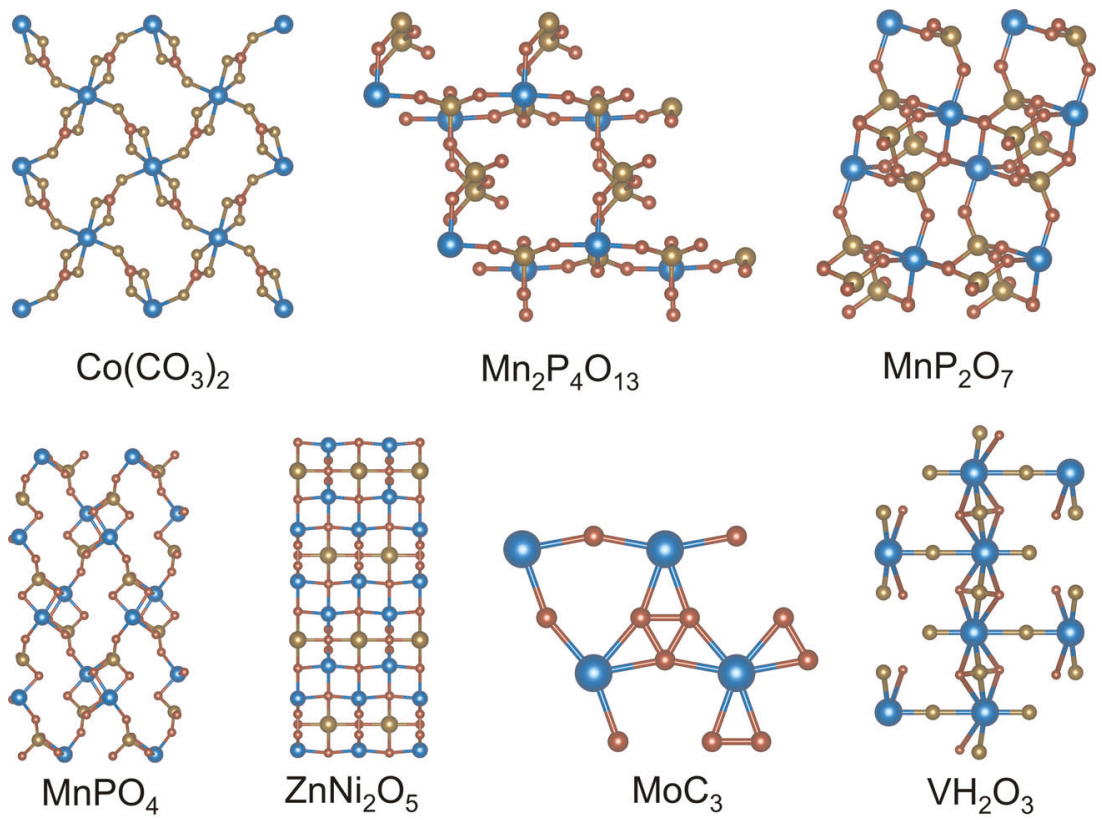

$\mathrm{MnP}_{2} \mathrm{O}_{7}$

$$
\mathrm{Mn}_{2} \mathrm{P}_{4} \mathrm{O}_{13}
$$
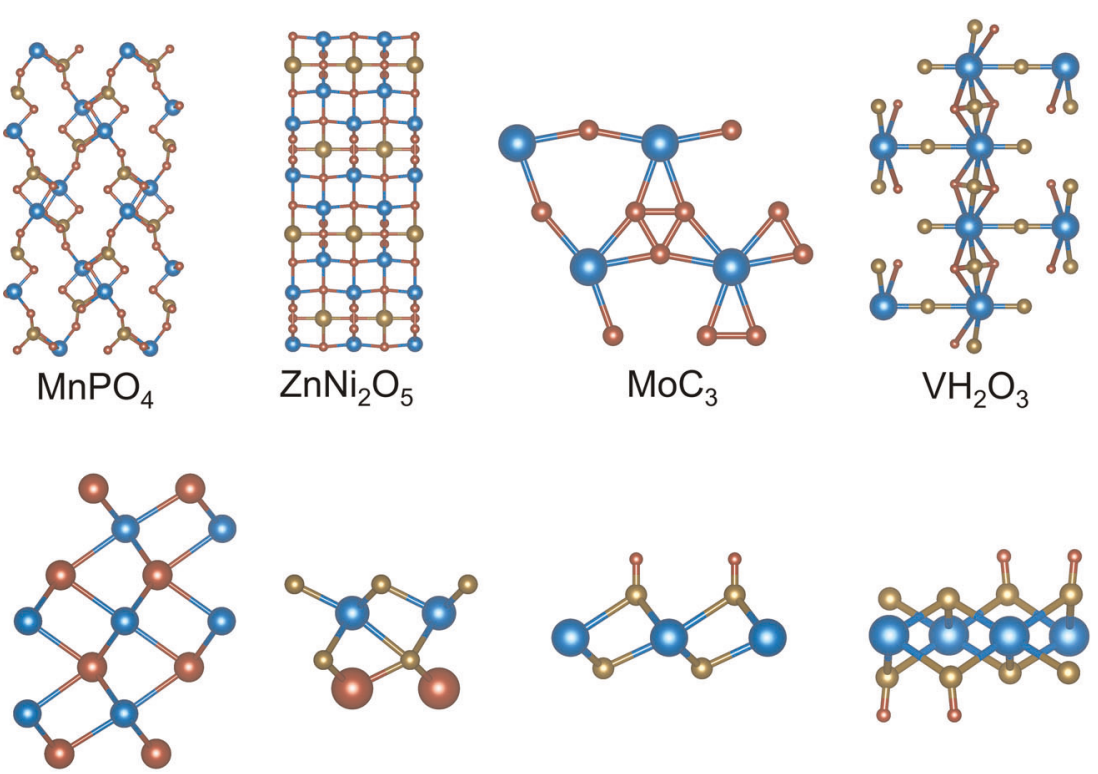

$$
\mathrm{Cr}_{3} \mathrm{Te}_{4}
$$

$\mathrm{LiCrO}_{2}$

$\mathrm{CrHO}_{2}$ P $3 \mathrm{~m} 1$

$\mathrm{CrHO}_{2} \mathrm{P}_{2}{ }_{1 / \mathrm{c}}$

Fig. 3 Prototypes of unique test data. The unique prototypes of the test data which have not been covered in Fig. 2. Note that for visualization purposes, top view of the materials in the 1st and 2nd row has been shown, but the third row contains all side-views. The same colour convention as Fig. 2 have been used.

a

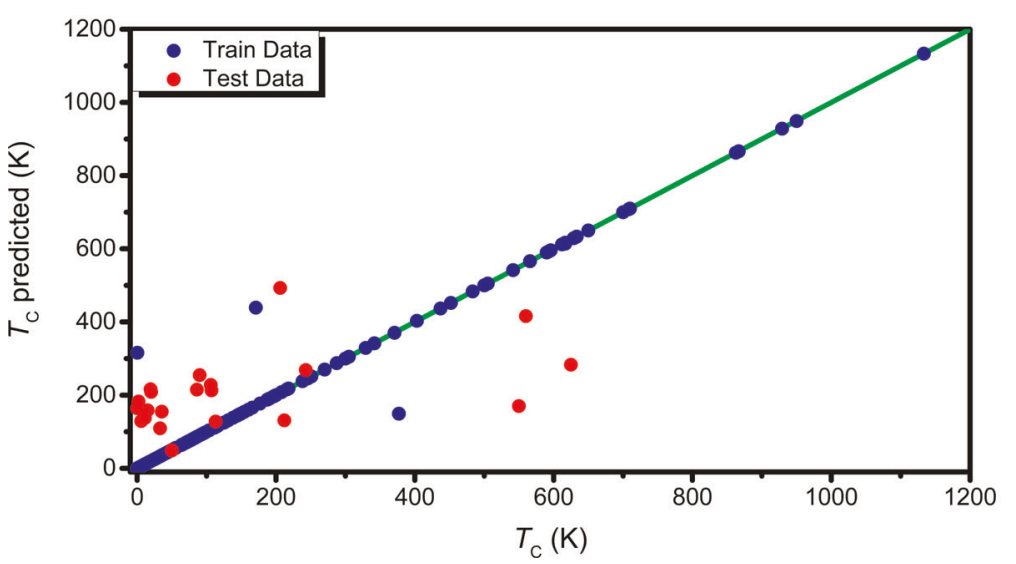

b

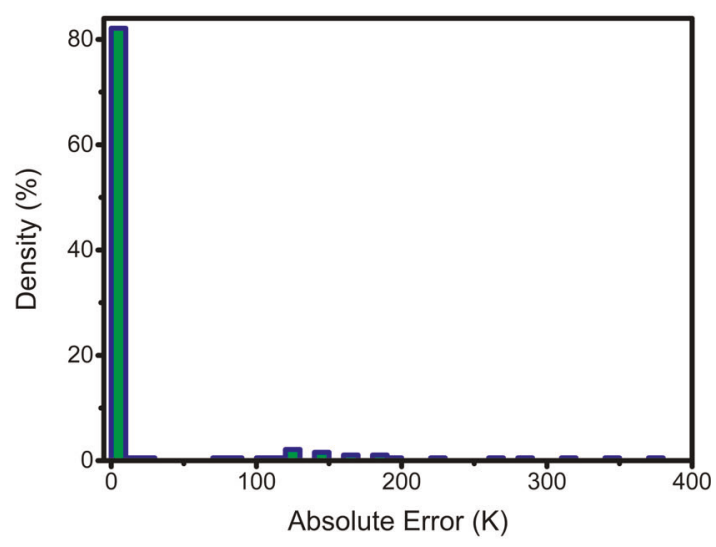

Fig. 4 Accuracy of the machine learning model. a plot of DFT-MC calculated $T_{\mathrm{C}}$ and machine learning predicted $T_{\mathrm{C}}$ for all the train as well as test samples. The green line represents a perfectly accurate prediction line. $\mathbf{b}$ distribution (histogram plot) of the absolute error of the machine learning prediction for test and train data combined.

Violation of Goodenough-Kanamori rule

The classical Goodenough-Kanamori semi-empirical rules ${ }^{19,20}$ for magnetic materials essentially states that when the magnetic-ion- anion-magnetic-ion bond angle is close to $180^{\circ}$, a strong AFM structure due to superexchange should prevail, whereas if this angle is close to $90^{\circ}$, the material should show ferromagnetism. 
However, in this work, we notice quite a few violations of this rule. We have tabulated these bond angles for all the train and test materials in Supplementary Spreadsheet 2 and the apparent violation cases are marked in red. The most significant violation of the Goodenough-Kanamori criterion can be seen in the strong high- $T_{C} \quad F M$ material $\mathrm{CrO}_{2} \quad \mathrm{P} 4 / \mathrm{mmm}$, where the cation-anion-cation bond angle is precisely $180^{\circ}$. Although a full investigation is out of the scope of this work, it appears that these postulates were developed for bulk materials and are failing here because of highly covalent bonds of 2DFM materials. Especially $\mathrm{CrO}_{2} \_\mathrm{P} 4 / \mathrm{mmm}$ exhibits a highly planar structure, and thus should manifest highly covalent bonds. However, this material seems to be dynamically unstable and possesses a much higher total energy compared to the experimentally available bulk phase which might make it unlikely to be experimentally synthesized ${ }^{9}$.

\section{High-temperature structural stability}

According to the thermodynamic stability classification in C2DB, we choose three highly stable high- $T_{C}$ materials, namely CrIN_Pmmm, $\mathrm{RhCl}_{2} \mathrm{C} 2 / \mathrm{m}$, and $\mathrm{Mn}_{2} \mathrm{H}_{2} \mathrm{CO}_{2} \mathrm{P}-3 \mathrm{~m} 1$ along with newfound $\mathrm{ZnNi}_{2} \mathrm{O}_{5}$ Pmmn for further structural stability evaluation at high temperatures. An ab-initio molecular dynamics (AIMD) run at $400 \mathrm{~K}$ for a total of $6.5 \mathrm{ps}$ is conducted for this. Although $\mathrm{CrIN}, \mathrm{RhCl}_{2}$, and $\mathrm{ZnNi}_{2} \mathrm{O}_{5}$ retain their crystal structure during this, albeit with less crystallinity, the $M X e n e \mathrm{Mn}_{2} \mathrm{H}_{2} \mathrm{CO}_{2}$ starts to melt away just after $3 \mathrm{ps}$, rendering it unsuitable for practical applications. Supplementary Figs 7-10 show the structural differences resulted from the MD runs.

\section{DISCUSSION}

In this study, we predict a total of $262 \mathrm{D}$ materials to have $T_{\mathrm{C}}$ beyond $400 \mathrm{~K}$. Many of these could be easily synthesizable, either by straight exfoliation from their bulk counterparts or by bottom-up chemical methods ${ }^{24,25}$. It is worth noting that, low thermodynamic stability does not necessarily mean the material would be unusable for practical applications. For instance, according to C2DB, Silicene is classified as a material with low thermodynamic stability. However, Silicene transistors have been demonstrated to work in room temperature ${ }^{35}$. Some of these materials screened by us even show dynamic instability. Again, many commonly used 2D materials, such as T-phase $\mathrm{MoS}_{2}$ showing dynamic instability ${ }^{36}$ in free-standing form stabilizes themselves on substrates through possible substrate interaction and even finds application in room-temperature devices ${ }^{37}$. Also, these materials can show charge density wave (CDW) characteristics and stabilize in a larger supercell with slight structural distortion ${ }^{7}$. However, CDW distortions can sometimes impact the magnetic order adversely ${ }^{38}$.

To summarize, using high-throughput automated codes and data-driven models, we thoroughly screen 2D materials databases and predict a host of 2DFM materials with high Curie point. With the emergence of novel synthesis techniques, these materials could indeed be of interest to experimentalists and engineers in terms of practical application in various devices. The ML model and the automated code developed in this work could find use in the community for rapid magnetic property prediction. The model complements the rigorous DFT-MC based code and if trained with sufficiently large datasets the model could eventually replace the code ${ }^{32}$. State-of-the-art software engineering enables us to achieve an optimal balance between rigor and computational efficiency, which is very important for reliable high-throughput material screening. As a result, we discover many important magnetic materials involving metals like Mo, $\mathrm{W}$ and $\mathrm{Ti}$ which have so far been ignored by heuristicbased formula-screening ${ }^{10}$.

\section{METHODS}

Spin configurations generation

As mentioned before, the python library pymatgen ${ }^{21}$ has been extensively used in this study for generating spin configurations, managing, and parsing input-output files and performing the neighbour mappings. The python module $\mathrm{ASE}^{39}$ has also been used to parse the input structure files. To ensure the reliability and coverage of the pymatgen generated spin configurations, we manually verify that the code-generated spin configuration set almost always includes all heuristics-based configurations reported in the literature ${ }^{9-14}$. Often, the code generates even more unexplored but symmetrically valid configurations that we leverage to include a higher number of neighbour interactions for a more accurate prediction of the $T_{\mathrm{C}}$. At the same time, we ignore ferrimagnetic configurations since these are usually energetically highly unstable as well as asymmetric. For instance, in contrast to the previous report ${ }^{10}$, our $T_{C}$ prediction of $\mathrm{Cr}_{3} \mathrm{Te}_{4}$ is based on a larger number of symmetric $\mathrm{FM}$ and AFM configurations and thus seems to be much more accurate.

It is worth mentioning that since we cover such a huge variety of materials using an automated workflow, we use such values of various DFT and numerical parameters [see Supplementary Readme (readme file at OSF repository)], which would yield reasonable results for all materials. For instance, in case of $\mathrm{Crl}_{3}$, the default value of the parameter enum_prec = 0.001 (enum_precision_parameter in pymatgen) generates 3 configurations, where the Néel AFM configuration gets excluded (Supplementary Fig 1). But with enum_prec $=1 \mathrm{e}-7$, all 4 configurations can be generated. For the first case, we obtain $J_{1}=2.78$ and $J_{2}=0.43 \mathrm{meV} /$ link, while the second set gives us almost identical values of $J_{1}=2.82, J_{2}=0.41$ and $J_{3}=$ $0.009 \mathrm{meV} / \mathrm{link}$ and in both cases, we obtain the same $T_{\mathrm{c}}$. The Néel AFM solution turns out to be the most energetically unfavourable state and gets truncated $^{18}$ by pymatgen-enumlib with default settings. For this highthroughput study, we find the default values to be accurate enough for our one-fits-all scheme. However, for focused studies on some specific material one might want to obtain extremely accurate results and thus might need to tune the parameters a little.

\section{DFT parameters}

Because the energy differences between various configurations for magnetism calculations could be as low as $\approx \mu \mathrm{eV}$, the computations need to be performed with high accuracy. We use heavily modified versions of predefined configurations 'MPRelaxSet', 'MPStaticSet' and 'MPSOCSet' available in pymatgen for relaxations, static runs and MAE calculations. These modifications, as well as other details of DFT, are highlighted below.

Spin-polarized DFT calculations are carried out using generalized gradient approximation (GGA) as implemented in the code VASP ${ }^{40}$ with projector augmented-wave (PAW $)^{41}$ method using the Perdew-Burke-Ernzenhof (PBE) ${ }^{42}$ exchange-correlation functional. Along with the CPU version, The GPU port ${ }^{43}$ of VASP has been used extensively. For all calculations, a correction on the strongly correlated d-shell electrons $(\mathrm{GGA}+U)$ is applied using the Dudare ${ }^{44}$ formulation. The default value of the cut-off energy $(520 \mathrm{eV})$ is used which proved to be sufficiently large. For relaxations, the default reciprocal density of $64 \AA^{-3}$ is employed whereas for all collinear and non-collinear static runs a much denser reciprocal density of $300 \AA^{-3}$ has been used. Electronic convergence is set to be attained when the difference in energy of successive electronic steps becomes less than $10^{-6} \mathrm{eV}$, whereas the structural geometry is optimized until the maximum Hellmann-Feynman force on every atom falls below $0.01 \mathrm{eV} / \mathrm{A}$. For the high-precision MAE calculations, a stricter electronic convergence criterion of $10^{-8} \mathrm{eV}$ is imposed. A large vacuum space of $>25 \AA$ in the direction of $\mathbf{c}$ is applied to avoid any spurious interaction between periodically repeated layers. The Bader charge and magnetization analysis are performed using the code developed by the Henkelman group ${ }^{45}$, where charge densities generated from DFT static runs are used as inputs. These Bader partitioned magnetic moments have been used as the local magnetic moments of the magnetic elements. All crystal structure images are generated using the tool VESTA ${ }^{46}$.

For metals, $\mathrm{Co}, \mathrm{Cr}, \mathrm{Fe}, \mathrm{Mn}, \mathrm{Mo}, \mathrm{Ni}, \mathrm{V}$ and $\mathrm{W}$, the effective $U$ values have been taken from the Materials Project (https://wiki.materialsproject.org/ GGA\%2BU_calculations\#Calibration_of_U_values) where these effective $U$ values have been calibrated by performing a fitting to experimental binary formation enthalpies ${ }^{47}$. This is an established practice and has been used in similar high-throughput screening studies before ${ }^{10}$. We also find that the application of an effective $U$ is essential to perform accurate DFT calculations on materials with "low" magnetization (materials containing $\mathrm{Nb}, \mathrm{Sc}, \mathrm{Ru}, \mathrm{Rh}, \mathrm{Pd}, \mathrm{Cu}, \mathrm{Os}, \mathrm{Ti}, \mathrm{Zr}, \mathrm{Re}, \mathrm{Hf}, \mathrm{Pt}$, and $\mathrm{La}$ ) as the AFM solutions 
become difficult to obtain for these materials without a proper effective $U$. In these cases, for a specific metal, first the effective $U$ values are obtained using the linear-response approach ${ }^{30}$ using $3 \times 3$ supercells for a few materials containing the element and then the average value of these effective $U$ is taken as the final effective $U$. A complete list of effective $U$ values (in eV) used in this study, as well as the DFT parameters imposed can be found in Supplementary Code 1 (e2e.py at OSF repository).

It is worth noting that, these effective $U$ values depend on the element type, charge state and coordination mode of magnetic species in a certain material, which implies that the value of $U$ is quite material-specific and should be determined carefully for accurate predictions. Thus, we also calculate the material-specific $U$ values for the most promising 26 materials with predicted high- $T_{C}$ using the linear-response approach ${ }^{30}$. We observe that the $U$ values obtained from the linear-response method can be quite different from the high-throughput $U$ values we have used so far (see Sheet 3 of Supplementary Spreadsheet 2). However, the computational budget of the linear-response method is excessively high to be adopted for high-throughput material screening.

\section{Hamiltonian fitting}

The coupling constant $(J)$ values are fitted using the collinear energy values of different FM and AFM spin configurations. However, for a lot of cases, when the energies of all configurations are taken, the determinant of the system of equations becomes zero which makes the set of equations unsolvable. As an automated remedy for these problems, again a fitting is tried omitting the most unstable AFM configuration and using one less neighbour than before. This process is repeated until a set of physically meaningful solutions is found, or the code runs out of configurations to fit. The anisotropic constant $(k)$ values are fitted using the non-collinear energy values with spins oriented in different directions ([100], [010], [110], [001]).

Despite our best efforts, calculations for 261 materials (out of the 786 materials classified as FM in (2DB) had to be cancelled because of the following reasons: (1) pymatgen could not recognize the symmetry and could generate only one configuration, (2) material turned out to be nonmagnetic after DFT calculations, (3) severe convergence issues occurred during DFT calculations, (4) AFM configurations could not be retained even after application of proper $U$ and manual tuning of parameters, (5) the Hamiltonian could not be fitted properly, (6) phase change of crystal structure after relaxation.

\section{Monte Carlo simulation}

To study ferromagnetic (FM) to paramagnetic (PM) transition in these monolayer materials, Monte Carlo (MC) simulations of the Heisenberg model have been performed using the Metropolis algorithm with singlespin update scheme ${ }^{48}$. To eliminate the size effects, a $50 \times 50$ supercell containing 2500-8000 sites has been used to simulate the system. Total $10^{5}$ Monte Carlo steps have been performed for each temperature, while the results from the first $10^{4}$ steps have been discarded, as the system is allowed to equilibrate (thermalize) during this time. The final values of magnetization and susceptibility are calculated as the average over the last $9 \times 10^{4}$ MC steps for each temperature.

\section{Software engineering}

We develop the complete end-to-end code in python to take advantage of pymatgen. However, python being an interpreted language, the $M C$ simulations turned out to be excessively slow, especially with high coordination numbers and inclusion of higher neighbours, which made the code unsuitable for the high-throughput study. As a remedy to this problem, we decide to use python-based just-in-time (JIT) compiler numba ${ }^{49}$ which compiles specific decorated python modules at the first encounter to lowlevel instructions, and when these modules are repeatedly called, the compiled version is used which makes the code extremely fast. However, the trade-off is, a lot of powerful functions and the coding flexibility offered by python (like heterogeneous data structures, appending to a list) cannot be successfully compiled and significant software engineering, as well as timing and cost-benefit analyses, are required to achieve an optimal code. GPU acceleration for neighbour-mapping of large lattices (2500-8000 sites) has also been implemented, which on a CPU must be done serially and takes a lot more time.

The engineered code was optimized to such an extent that the whole study could be performed using even a workstation-grade machine, albeit with GPU acceleration. A video of real-time execution of the code, where the $T_{\mathrm{C}}$ of four materials $\left(\mathrm{Crl}_{3}, \mathrm{Cr}_{2} \mathrm{Ge}_{2} \mathrm{Te}_{6}, \mathrm{MnSe}_{2}\right.$, and $\left.\mathrm{MoC}_{3}\right)$ are being calculated in parallel in a single-CPU (18 cores), three-GPU enabled workstation within $\approx 10$ hour, can be found at https://youtu.be/HJkR$03 \mathrm{OzBl}$. At the same time excellent scalability is observed, when the code is executed on a high performance computing node (https://youtu.be/ GQaFfm29LR4).

\section{Machine learning}

The python libraries automatminer and matminer ${ }^{33}$ have been used to featurize the datasets and search for optimal ML pipeline for the FM/AFM classification problem as well as the $T_{C}$ predicting regression problem. Dataset cleaning and feature reduction are handled by automatminer. Then, various pre-processing algorithms along with a host of commonly used ML models employed for materials science problems ${ }^{31}$ for small to moderate datasets have been searched by the autoML library TPOT ${ }^{34}$, such as naive Bayesian, decision tree, extra trees, random forest, gradient boosting, k-neighbors, linear SVC and logistic regression for classification and elastic net CV, decision tree, extra trees, random forest, gradient boosting, k-neighbors, lasso lars CV, ridge CV and linear SVR for regression. Also, the hyperparameters are tuned at the same time. A full list of preprocessing algorithms, ML models and hyperparameters searched can be found at https://github.com/hackingmaterials/automatminer/blob/master/ automatminer/automl/config/tpot_configs.py. It is worth noting that TPOT uses the ML library scikit-learn ${ }^{50}$ for the $M L$ as well as the featureengineering models. For each autoML search, more than 60,000 pipelines have been explored.

The generic python codes using scikit-learn for the best pipelines for both FM/AFM classification and $T_{C}$ regression have been given in Supplementary Codes 2 and 3 (TPOT *.py at OSF repository). For the former, a combination of SelectPercentile, MaxAbsScaler, and ExtraTreesClassifier turns out to be the best pipeline, whereas for the latter it is a combination of SelectPercentile, ZeroCount, and GradientBoostingRegressor. The list of features used for these problems can be found in Supplementary Logs 1 and 2 (*_digest at OSF repository). Moreover, the pickled pipelines have also been provided in Supplementary ML Pipes 1 and 2 (*.pipe at OSF repository) which can be loaded into automatminer to make predictions on any dataset. The Supplementary Readme file provides detailed instructions on how to use the Supplementary codes and pipes.

\section{AIMD simulations}

For the chosen materials, to minimize the temperature oscillations a large supercell containing $\geq 144$ atoms has been constructed to run the AIMD on. Because of severe convergence issues, non-spin-polarized DFT calculations are performed with Gamma-point only sampling which can be sufficient to determine structural stability. A canonical ensemble (NVT) is used and a Nosé-Hoover thermostat ${ }^{51,52}$ at $400 \mathrm{~K}$ is employed. The simulations run for $6.5 \mathrm{ps}$ with a $2 \mathrm{fs}$ time step. The crystal structures of the tested materials after the simulation can be seen in Supplementary Figs 7-10.

\section{DATA AVAILABILITY}

The authors declare that the main data supporting the findings of this study are available within the paper and its Supplementary files, the OSF repository (https://osf. io/6ebjp/) and other open online resources. Other relevant data are available from the corresponding author upon reasonable request.

\section{CODE AVAILABILITY}

All relevant python codes and pickled $\mathrm{ML}$ pipelines are provided in the OSF Repository (https://osf.io/6ebjp/). Also, the end-to-end code for Curie point determination (e2e.py) is available at GitHub (https://github.com/NSDRLIISc/e2e), which we plan to update periodically.

Received: 3 December 2019; Accepted: 10 March 2020; Published online: 08 April 2020

\section{REFERENCES}

1. Huang, B. et al. Layer-dependent ferromagnetism in a van der Waals crystal down to the monolayer limit. Nature 546, 270-273 (2017).

2. Gong, $C$. et al. Discovery of intrinsic ferromagnetism in two-dimensional van der Waals crystals. Nature 546, 265 (2017). 
3. Feng, Y. P. et al. Prospects of spintronics based on 2D materials. Wiley Interdiscip. Rev. Comput. Mol. Sci. 7, e1313 (2017).

4. Farooq, M. U. \& Hong, J. Switchable valley splitting by external electric field effect in graphene/ $\mathrm{Crl}_{3}$ heterostructures. npj 2D Mater. Appl. 3, 3 (2019).

5. Soumyanarayanan, A., Reyren, N., Fert, A. \& Panagopoulos, C. Emergent phenomena induced by spin-orbit coupling at surfaces and interfaces. Nature 539, 509-517 (2016).

6. Mermin, N. D. \& Wagner, H. Absence of ferromagnetism or antiferromagnetism in one- or two-dimensional isotropic Heisenberg models. Phys. Rev. Lett. 17, 1133-1136 (1966).

7. Bonilla, M. et al. Strong room-temperature ferromagnetism in $\mathrm{VSe}_{2}$ monolayers on van der Waals substrates. Nat. Nanotechnol. 13, 289-293 (2018).

8. O'Hara, D. J. et al. Room temperature intrinsic ferromagnetism in epitaxial manganese selenide films in the monolayer limit. Nano Lett. 18, 3125-3131 (2018).

9. van Gog, H. et al. Thermal stability and electronic and magnetic properties of atomically thin 2D transition metal oxides. npj 2D Mater. Appl. 3, 18 (2019).

10. Zhu, Y., Kong, X., Rhone, T. D. \& Guo, H. Systematic search for two-dimensional ferromagnetic materials. Phys. Rev. Mater. 2, 81001 (2018).

11. Kumar, $\mathrm{H}$. et al. Tunable magnetism and transport properties in nitride MXenes. ACS Nano 11, 7648-7655 (2017).

12. Jiang, Z., Wang, P., Xing, J., Jiang, X. \& Zhao, J. Screening and design of Novel 2D ferromagnetic materials with high curie temperature above room temperature. ACS Appl. Mater. Interfaces 10, 39032-39039 (2018).

13. Zhuang, H. L., Xie, Y., Kent, P. R. C. \& Ganesh, P. Computational discovery of ferromagnetic semiconducting single-layer $\mathrm{CrSnTe}_{3}$. Phys. Rev. B 92, 35407 (2015).

14. Miao, N., Xu, B., Zhu, L., Zhou, J. \& Sun, Z. 2D intrinsic ferromagnets from van der Waals antiferromagnets. J. Am. Chem. Soc. 140, 2417-2420 (2018).

15. Mounet, N. et al. Two-dimensional materials from high-throughput computational exfoliation of experimentally known compounds. Nat. Nanotechnol. 13, 246-252 (2018).

16. Haastrup, S. et al. The Computational 2D Materials Database: high-throughput modeling and discovery of atomically thin crystals. 2D Mater. 5, 42002 (2018).

17. Zhou, J. et al. 2DMatPedia, an open computational database of two-dimensional materials from top-down and bottom-up approaches. Sci. Data 6, 86 (2019).

18. Horton, M. K., Montoya, J. H., Liu, M. \& Persson, K. A. High-throughput prediction of the ground-state collinear magnetic order of inorganic materials using density functional theory. npj Comput. Mater. 5, 64 (2019).

19. Goodenough, J. B. An interpretation of the magnetic properties of the perovskitetype mixed crystals $\mathrm{La}_{1-x} \mathrm{Sr}_{x} \mathrm{CoO}_{3-\lambda}$. J. Phys. Chem. Solids 6, 287-297 (1958).

20. Kanamori, J. Superexchange interaction and symmetry properties of electron orbitals. J. Phys. Chem. Solids 10, 87-98 (1959).

21. Ong, S. P. et al. Python Materials Genomics (pymatgen): a robust, open-source python library for materials analysis. Comput. Mater. Sci. 68, 314-319 (2013).

22. Torelli, D. \& Olsen, T. Calculating critical temperatures for ferromagnetic order in two-dimensional materials. 2D Mater. 6, 15028 (2018).

23. Ashton, M. et al. Two-dimensional intrinsic half-metals with large spin gaps. Nano Lett. 17, 5251-5257 (2017).

24. Zhang, J. et al. Janus monolayer transition-metal dichalcogenides. ACS Nano 11, 8192-8198 (2017).

25. Sreepal, V. et al. Two-dimensional covalent crystals by chemical conversion of thin van der Waals materials. Nano Lett. 19, 6475-6481 (2019).

26. Fei, Z. et al. Two-dimensional itinerant ferromagnetism in atomically thin $\mathrm{Fe}_{3} \mathrm{GeTe}_{2}$. Nat. Mater. 17, 778-782 (2018)

27. Zhuang, H. L., Kent, P. R. C. \& Hennig, R. G. Strong anisotropy and magnetostriction in the two-dimensional Stoner ferromagnet $\mathrm{Fe}_{3} \mathrm{GeTe}_{2}$. Phys. Rev. B 93, 134407 (2016).

28. Wang, X. et al. Raman spectroscopy of atomically thin two-dimensional magnetic iron phosphorus trisulfide ( $\mathrm{FePS}_{3}$ ). Cryst. 2D Mater. 3, 31009 (2016).

29. Kim, K. et al. Suppression of magnetic ordering in XXZ-type antiferromagnetic monolayer $\mathrm{NiPS}_{3}$. Nat. Commun. 10, 345 (2019).

30. Cococcioni, M. \& de Gironcoli, S. Linear response approach to the calculation of the effective interaction parameters in the LDA+U method. Phys. Rev. B 71, 35105 (2005).

31. Schmidt, J., Marques, M. R. G., Botti, S. \& Marques, M. A. L. Recent advances and applications of machine learning in solid-state materials science. npj Comput. Mater. 5, 83 (2019).

32. Nelson, J. \& Sanvito, S. Predicting the Curie temperature of ferromagnets using machine learning. Phys. Rev. Mater. 3, 104405 (2019).

33. Ward, L. et al. Matminer: an open source toolkit for materials data mining. Comput. Mater. Sci. 152, 60-69 (2018).

34. Olson, R. S., Bartley, N., Urbanowicz, R. J. \& Moore, J. H. Evaluation of a tree-based pipeline optimization tool for automating data science. In Proc Genetic and Evolutionary Computation Conference 2016 (ed. Friedrich, T.) 485-492 (ACM, 2016).
35. Tao, L. et al. Silicene field-effect transistors operating at room temperature. Nat. Nanotechnol. 10, 227-231 (2015).

36. Saha, D. \& Mahapatra, S. Atomistic modeling of the metallic-to-semiconducting phase boundaries in monolayer $\mathrm{MoS}_{2}$. Appl. Phys. Lett. 108, 253106 (2016).

37. Kappera, R. et al. Phase-engineered low-resistance contacts for ultrathin $\mathrm{MoS}_{2}$ transistors. Nat. Mater. 13, 1128 (2014).

38. Zheng, F., Zhou, Z., Liu, X. \& Feng, J. First-principles study of charge and magnetic ordering in monolayer $\mathrm{NbSe}_{2}$. Phys. Rev. B 97, 81101 (2018).

39. Hjorth Larsen, A. et al. The atomic simulation environment-a Python library for working with atoms. J. Phys. Condens. Matter 29, 273002 (2017).

40. Kresse, G. \& Furthmüller, J. Efficiency of ab-initio total energy calculations for metals and semiconductors using a plane-wave basis set. Comput. Mater. Sci. 6, 15-50 (1996).

41. Kresse, G. \& Joubert, D. From ultrasoft pseudopotentials to the projector augmented-wave method. Phys. Rev. B 59, 1758-1775 (1999).

42. Perdew, J. P., Burke, K. \& Ernzerhof, M. Generalized Gradient Approximation Made Simple. Phys. Rev. Lett. 77, 3865-3868 (1996).

43. Hacene, M. et al. Accelerating VASP electronic structure calculations using graphic processing units. J. Comput. Chem. 33, 2581-2589 (2012).

44. Dudarev, S. L., Botton, G. A., Savrasov, S. Y., Humphreys, C. J. \& Sutton, A. P. Electron-energy-loss spectra and the structural stability of nickel oxide: An LSDA+U study. Phys. Rev. B 57, 1505-1509 (1998).

45. Hu, L. et al. Two-dimensional materials from high-throughput computational exfoliation of experimentally known compounds. Phys. Rev. B 6, 1-7 (2017).

46. Momma, K. \& Izumi, F. VESTA3 for three-dimensional visualization of crystal, volumetric and morphology data. J. Appl. Crystallogr 44, 1272-1276 (2011).

47. Wang, L., Maxisch, T. \& Ceder, G. Oxidation energies of transition metal oxides within the GGA+U framework. Phys. Rev. B 73, 195107 (2006).

48. Vatansever, E. et al. Strain effects on electronic and magnetic properties of the monolayer $\mathrm{a}-\mathrm{RuCl}_{3}$ : A first-principles and Monte Carlo study. J. Appl. Phys. 125, 83903 (2019).

49. Lam, S. K., Pitrou, A. \& Seibert, S. Numba: a LLVM-based Python JIT Compiler. In Proc. 2nd Workshop on the LLVM Compiler Infrastructure in HPC (ed. Finkel, H.) 7:1-7:6 (ACM, 2015).

50. Pedregosa, F. et al. Scikit-learn: Machine Learning in Python. J. Mach. Learn. Res. 12, 2825-2830 (2011).

51. Nosé, S. A unified formulation of the constant temperature molecular dynamics methods. J. Chem. Phys. 81, 511-519 (1984).

52. Hoover, W. G. Canonical dynamics: equilibrium phase-space distributions. Phys. Rev. A 31, 1695-1697 (1985).

\section{ACKNOWLEDGEMENTS}

The authors are grateful to Dr. Naihua Miao, Beihang University and Prof. Ümit Akıncı, Dokuz Eylül University for several insightful discussions. Authors also thank Abdul Samadh, Manipal University, and Kota Hemanth, IIT Delhi, for their help with software engineering and machine learning. The authors thank Supercomputer Education and Research Centre (SERC), Indian Institute of Science (IISC) Bangalore, for CPU based computations. The GPU computing nodes used in this study were procured through the research funding from the Department of Science and Technology (DST), Government of India, under grant number DST/TMD/MES/2K18/28.

\section{AUTHOR CONTRIBUTIONS}

A.K. developed the automated end-to-end code, implemented it on CPU and GPU based architectures, developed the ML models and analysed the data. M.K. scanned the materials using the automated code, collected and verified the results. S.M. conceived the problem statement and overall supervised the work. All authors contributed to the writing.

\section{COMPETING INTERESTS}

The authors declare no competing interests.

\section{ADDITIONAL INFORMATION}

Supplementary information is available for this paper at https://doi.org/10.1038/ s41524-020-0300-2.

Correspondence and requests for materials should be addressed to A.K.

Reprints and permission information is available at http://www.nature.com/ reprints 
Publisher's note Springer Nature remains neutral with regard to jurisdictional claims in published maps and institutional affiliations.

(c) Open Access This article is licensed under a Creative Commons Attribution 4.0 International License, which permits use, sharing, adaptation, distribution and reproduction in any medium or format, as long as you give appropriate credit to the original author(s) and the source, provide a link to the Creative
Commons license, and indicate if changes were made. The images or other third party material in this article are included in the article's Creative Commons license, unless indicated otherwise in a credit line to the material. If material is not included in the article's Creative Commons license and your intended use is not permitted by statutory regulation or exceeds the permitted use, you will need to obtain permission directly from the copyright holder. To view a copy of this license, visit http://creativecommons. org/licenses/by/4.0/.

c) The Author(s) 2020 